\title{
Validation of handheld meters to measure blood L-lactate concentration in dairy cows and calves
}

\author{
O. Burfeind and W. Heuwieser ${ }^{1}$ \\ Clinic for Animal Reproduction, Faculty of Veterinary Medicine, Freie Universität Berlin, Koenigsweg 65, 14163 Berlin, Germany
}

\begin{abstract}
In cattle, blood lactate was measured in various conditions such as parturition and dystocia. To our knowledge, to date, no handheld device has been validated for the use in cows and only one handheld device was validated for the use in calves. When determining plasma lactate concentrations blood samples have to be processed carefully. Sodium fluoride was recommended to inhibit glycolysis and to stabilize plasma lactate concentrations during transport. However, its effect on measurements conducted with electrochemical meters has not been studied. The objectives of 3 experiments were to study factors influencing measures of L-lactate in dairy cows (e.g., different anticoagulants, different methods) and to validate a handheld device (Lactate Scout, SensLab GmbH, Leipzig, Germany) to determine L-lactate concentration in dairy cows and calves. In a first approach, blood samples from 49 cows were analyzed by 2 different laboratories. Measures of L-lactate concentration were correlated between the different laboratories in both lithium heparin plasma $(\mathrm{r}=0.98)$ and sodium fluoride plasma $(\mathrm{r}=0.99)$. In a second approach, these samples were analyzed using 3 methods [Lactate Scout, Biosen C_line (EKF Diagnostics GmbH, Barleben, Germany), and commercial laboratory]. Concentrations of L-lactate measured in lithium heparin did not differ when analyzed with the Lactate Scout $(0.99 \pm 0.35 \mathrm{mmol} / \mathrm{L})$, the Biosen C_line $(0.81 \pm 0.26 \mathrm{mmol} / \mathrm{L})$, or the laboratory $(1.0 \pm 0.36$ $\mathrm{mmol} / \mathrm{L})$. Concentrations of L-lactate measured in sodium fluoride, however, were higher when analyzed with the Lactate Scout $(1.85 \pm 0.66 \mathrm{mmol} / \mathrm{L})$ compared with those measured with the Biosen C_line (0.92 \pm $0.37 \mathrm{mmol} / \mathrm{L})$ and by the commercial laboratory $(0.72$ $\pm 0.45 \mathrm{mmol} / \mathrm{L})$. In the second and third experiments, blood samples from 173 cows and 106 calves were analyzed using the 3 methods (Lactate Scout, Biosen C_line, and commercial laboratory). L-Lactate concentrations measured with the 3 methods were correlated
\end{abstract}

Received January 10, 2012.

Accepted June 28, 2012

${ }^{1}$ Corresponding author: w.heuwieser@fu-berlin.de (cows: Lactate Scout vs. Biosen C_line: $\mathrm{r}=0.97$, Lactate Scout vs. laboratory 1: $\mathrm{r}=0.98$, Biosen C_line vs. laboratory 1: $\mathrm{r}=0.99$; calves: Lactate Scout vs. Biosen C_line: $\mathrm{r}=0.97$, Lactate Scout vs. laboratory 1: $\mathrm{r}=0.98$, Biosen C_line vs. laboratory 1: $\mathrm{r}=0.99)$. In conclusion, Lactate Scout and Biosen C_line measure blood L-lactate concentrations reliably compared with a commercial laboratory as the reference method in dairy cows and calves. However, attention needs to be paid to the choice of anticoagulant used in sample collection. Key words: dairy cow, calf, L-lactate, handheld meter

\section{INTRODUCTION}

Lactate in blood plasma is the result of anaerobic glycolysis and is formed when pyruvate is converted by lactate dehydrogenase into lactic acid (Lagutchik et al., 1996). Major lactate producers are skeletal muscle and gut tissues (Arieff and Graf, 1987). Concentration of blood lactate increases in cases of local or systemic hypoperfusion (Hughes et al., 1999). Hyperlactemia in humans and dogs is defined as increased blood lactate concentrations of 2 to $5 \mathrm{mmol} / \mathrm{L}$ (Mizock, 1987) and $>5 \mathrm{mmol} / \mathrm{L}$ (Hughes et al., 1999), respectively.

In cattle, lactate is measured in various conditions such as parturition and dystocia. Several studies have demonstrated higher concentrations of lactate for calves delivered after calving assistance compared with calves born spontaneously (Diesch et al., 2004; Sorge et al., 2009). Diesch et al. (2004) found higher plasma lactate concentrations in Friesian calves born with assistance $(6.3 \pm 0.76 \mathrm{mmol} / \mathrm{L})$ than in calves born without assistance $(4.7 \pm 0.76 \mathrm{mmol} / \mathrm{L})$ and in crossbred calves (Friesian $\times$ Angus) born with assistance $(9.1 \pm 0.87$ $\mathrm{mmol} / \mathrm{L})$ than in calves born without assistance (6.5 $\pm 0.62 \mathrm{mmol} / \mathrm{L})$. Furthermore, a negative correlation between a modified APGAR score (a scoring system to describe the vitality of a newborn calf: appearance, pulse, grimace, activity, respiration) and blood lactate concentration was demonstrated (Sorge et al., 2009). However, wide variability exists in lactate concentration in calves with different APGAR scores. In another approach, researchers evaluated blood lactate concentrations of cows and calves during the second 
stage of labor via puncture of the vena metacarpalis volaris superficialis every $10 \mathrm{~min}$. Lactate concentration increased during the birth process and was highest immediately after birth (Mülling et al., 1979).

Relationships of blood lactate concentrations with certain diseases (e.g., neonatal diarrhea, respiratory diseases, displaced abomasum) have also been examined. Naylor (1987) studied the nature and severity of acidosis in calves with diarrhea to investigate the relationship between acidosis and dehydration of calves. Blood lactate concentrations were higher (lactic acidosis) in calves younger than $8 \mathrm{~d}$ than in older calves although older calves suffered more from a more severe metabolic acidosis (nonlactic acidosis). The author speculated that either impaired lactate clearance in young calves or low glycogen stores in older calves (that could have been anorectic for a longer time) might have contributed to the findings. Furthermore, the study revealed a correlation between blood lactate concentration and changes in hematocrit during the first $6 \mathrm{~h}$ of fluid therapy ( $\mathrm{r}$ $=0.55)$. The prognostic value of blood lactate concentrations was also demonstrated for bovine respiratory diseases (Coghe et al., 2000). Belgian White and Blue calves suffering from respiratory disease with a blood lactate concentration exceeding $4 \mathrm{mmol} / \mathrm{L}$ were more likely to die than calves with a blood lactate concentration $<4 \mathrm{mmol} / \mathrm{L}$. Among calves that died $(\mathrm{n}=22)$, only 2 had lactate concentrations $<4 \mathrm{mmol} / \mathrm{L}$, whereas among calves that survived $(\mathrm{n}=78)$, only 2 had lactate concentration $>4 \mathrm{mmol} / \mathrm{L}$. The authors concluded that blood lactate concentration might be useful to decide whether to treat or euthanize a calf with respiratory disease.

More recently, lactate measurements were utilized to measure physical fitness of pregnant and nonpregnant cows (Davidson and Beede, 2009). Cows were either trained or not trained and their physical fitness tested on a treadmill on d 0,30 , and 60 after the beginning of training. After $60 \mathrm{~d}$ of training, increases in plasma lactate concentrations were smaller for exercised compared with nonexercised cows in both pregnant and nonpregnant cows (Davidson and Beede, 2009).

In human medicine, electronic handheld blood glucose and ketone measuring systems are widely used for diabetes monitoring (Guerci et al., 2005). Electrochemical handheld devices are also becoming more common for an immediate determination of blood metabolites in animals. Such devices were used to determine BHBA in cattle (Iwersen et al., 2009) and glucose in cats (Wess and Reusch, 2000a) and dogs (Wess and Reusch, 2000b). Handheld lactate analyzers were validated for use in dogs (Ferasin et al., 2007; Thorneloe et al., 2007; Tas et al., 2008) and foals (Castagnetti et al., 2010). In dogs and horses, lactate concentrations are mea- sured to evaluate exercise performance (Steiss et al., 2004; Campbell, 2011) and to assess the severity and prognosis of medical and surgical conditions (Lagutchik et al., 1998). Recently, a portable photometric lactate analyzer was validated in calves (28 d to 13 mo of age) with respiratory disease (Coghe et al., 2000). Although lactate concentrations measured with the device and the reference method were highly correlated $(\mathrm{r}=0.94)$, the portable apparatus slightly overestimated plasma lactate concentrations.

Glycolysis continues after blood collection and thus lactate concentration increases (Astles et al., 1994). Therefore, sodium fluoride alone or in combination with potassium oxalate was recommended to inhibit glycolysis and stabilize plasma lactate concentrations during transport (Astles et al., 1994). A determination of blood lactate concentrations immediately after blood collection with a handheld meter would be another option to avoid the bias caused by ongoing glycolysis. In previous validation trials, sodium fluoride and potassium oxalate were used (Castagnetti et al., 2010). However, their effect on measurements conducted with electrochemical meters has not been studied. From pilot work, we suspect that sodium fluoride interferes with lactate measurements of handheld meters.

Therefore the overall objectives of this study were (1) to test the accuracy of 2 commercial laboratories in measuring L-lactate from both lithium heparin and sodium fluoride plasma, (2) to study if a handheld meter measures higher values of L-lactate using sodium fluoride as anticoagulant than using lithium heparin, and (3) to validate a handheld meter (Lactate Scout, SensLab GmbH, Leipzig, Germany) for use in dairy cows and calves.

\section{MATERIALS AND METHODS}

Three experiments were conducted in July and August 2010 on a commercial dairy farm in SachsenAnhalt, Germany, housing approximately 1,200 Holstein-Friesian cows and 200 female calves. Animals were managed according to the guidelines set by the International Cooperation on Harmonization of Technical Requirements for Registration of Veterinary Medicinal Products (Hellmann and Radeloff, 2000). Cows were housed in a freestall barn with slotted floors, and cubicles were equipped with rubber mats. All calves were housed in straw-bedded boxes. Calves were singlehoused during the first $5 \mathrm{~d}$ of life and group-housed thereafter.

In the first experiment, plasma L-lactate concentrations measured by 2 commercial laboratories were compared using sodium fluoride and lithium heparin as anticoagulants. Furthermore, plasma L-lactate concentra- 
tions were analyzed using the same anticoagulants (i.e., sodium fluoride and lithium heparin) and 3 different methods: Lactate Scout, Biosen C_line (EKF Diagnostics GmbH, Barleben, Germany), and a wet chemistry analyzer (Cobas 6000, Roche Hitachi, Mannheim, Germany) in a commercial laboratory (laboratory 1). All methods used detected only the L-lactate isomer. The underlying method of the Lactate Scout was described previously (Castagnetti et al., 2010). In brief, $0.5 \mu \mathrm{L}$ of blood was applied on a test strip and L-lactate was detected by an enzymatic-amperometric system. The test-strip used L-lactate oxidase to catalyze the oxidation of L-lactate. The Biosen C_line used the same test principle: L-lactate was metabolized to pyruvate and hydrogen peroxide, which was detected by an electrode. The Biosen C_line is not a portable device but a stationary apparatus for laboratory use. Both commercial laboratories used wet chemistry analyzers from Roche Hitachi (laboratory 1: Cobas 6000; laboratory 2: Cobas C 501) and the same test kit (LACT2, Roche Hitachi). Again, L-lactate was metabolized to pyruvate and hydrogen peroxide. Instead of an electrochemical reaction as utilized with the Lactate Scout, in the kit, hydrogen peroxide reacted in the presence of peroxidase with 4-aminoantipyrine and $N$-ethyl- $N$-(2-hydroxy3-sulfopropyl)-3-methylaniline to form a red product. The intensity of the color is proportional to the lactate concentration in the sample (Castagnetti et al., 2010). Detailed information on the test characteristics of each method used is presented in Table 1.

Two blood samples were taken from the coccygeal vessels of 49 close-up cows (parity: $1.6 \pm 1.3$ ) with a evacuated collection tube system (Vacuette, Greiner Bio-One, Kremsmuenster, Austria). In the first sample, sodium fluoride and $\mathrm{K}_{3}$ EDTA were used as anticoagulants, whereas in the second sample, lithium heparin was used. Samples were analyzed using Lactate Scout and Biosen C_line within 5 min of collection. Furthermore, blood samples were stored on ice and centrifuged at $1,000 \times g$ for $10 \mathrm{~min}$ within $60 \pm 30 \mathrm{~min}$ of collection. Plasma was stored in 3 aliquots at $-20^{\circ} \mathrm{C}$ until analysis. Two aliquots each were sent to the commercial laboratories for analysis.

The objectives of the second and third experiments were to validate the Lactate Scout to measure L-lactate in the blood of dairy cows and calves using lithium heparin as anticoagulant. The gold standard was a plasma sample analyzed in a commercial laboratory (laboratory 1). Therefore, blood samples were taken from 173 cows (131 lactating cows: parity: $2.2 \pm 1.5$, DIM: $27 \pm 18 \mathrm{~d}$; 42 close-up cows: parity $1.9 \pm 1.3)$ and 106 calves (age: $17 \pm 12$ d). In calves, blood samples were taken from the jugular vein. Only 10 to 15 blood samples were taken at a time to reduce the time from sampling to analysis and centrifugation (20 $\pm 10 \mathrm{~min})$; samples were stored on ice until analysis. Blood samples were analyzed using the Lactate Scout and the Biosen C_line. Again, blood samples were centrifuged at 1,000 $\times g$ for 10 min and plasma stored at $-20^{\circ} \mathrm{C}$ until analysis in a commercial laboratory (laboratory 1 ).

Data were analyzed statistically with SPSS software (version 18.0, SPSS Inc., Munich, Germany). In experiment 1 , the relationship between plasma L-lactate concentrations measured by the 2 laboratories was determined with Pearson correlation coefficients for both anticoagulants, lithium heparin and sodium fluoride. The mean difference between laboratories was determined using a paired $t$-test. Similarly, the relationship and mean difference between L-lactate concentrations measured in lithium heparin plasma and sodium fluoride plasma were analyzed for each laboratory separately. Furthermore, L-lactate concentrations were determined in the blood samples with the Lactate Scout and Biosen C_line to calculate the differences between the different methods (Lactate Scout vs. Biosen C_line vs. commercial laboratory) and the 2 anticoagulants using a one-way ANOVA. Mean differences were determined with the post hoc test of Bonferroni.

In experiments 2 and 3 , relationships and differences between L-lactate concentrations measured with the Lactate Scout and Biosen C_line, and by the commercial laboratory were determined with Pearson cor-

Table 1. Test parameters for the different analytical methods ${ }^{1}$

Test parameter

\begin{tabular}{|c|c|c|c|c|}
\hline Method & $\begin{array}{l}\text { Analytical range } \\
(\mathrm{mmol} / \mathrm{L})\end{array}$ & Linearity $^{2}$ (n) & $\begin{array}{c}\text { Intraassay } \\
\text { CV }(\%)\end{array}$ & $\begin{array}{c}\text { Interassay } \\
\text { CV }(\%)\end{array}$ \\
\hline Lactate Scout & 0.5 to 25 & - & $\leq 5$ & $\leq 5$ \\
\hline Laboratory 2 & 0.2 to 15.5 & $\mathrm{y}=0.977 \mathrm{x}+0.043 \mathrm{mmol} / \mathrm{L}(69)$ & $\overline{<} 2.3$ & $\overline{<} 3.3$ \\
\hline
\end{tabular}

${ }^{1}$ Test parameters were delivered by the suppliers of the Lactate Scout (SensLab GmbH, Leipzig, Germany) and the Biosen C_line (EKF diagnostics GmbH, Barleben, Germany) and by laboratory 1 and laboratory 2.

${ }^{2}$ Linear regression over the analytical range calculated from human plasma samples, $\mathrm{n}=$ number of samples used for the calculation. 
relation and a one-way ANOVA. Mean differences were calculated with the post hoc test of Bonferroni.

A comparison of clinical measurements can be inappropriate using Pearson correlation. Therefore, the agreement between the different methods in each experiment was graphically analyzed using the method of Bland and Altman (1986) with MedCalc (version 12.0.3.0., MedCalc Software bvba, Mariakerke, Belgium).

\section{RESULTS}

L-Lactate concentrations in lithium heparin plasma (laboratory 1: $0.99 \pm 0.36 \mathrm{mmol} / \mathrm{L}$, laboratory 2: 1.03 $\pm 0.36 \mathrm{mmol} / \mathrm{L}, \mathrm{n}=49 ; P<0.01)$ and sodium fluoride plasma (laboratory 1: $0.71 \pm 0.44 \mathrm{mmol} / \mathrm{L}$, laboratory 2: $0.72 \pm 0.47 \mathrm{mmol} / \mathrm{L}, \mathrm{n}=49 ; P=0.71)$ were similar in both laboratories (Figure 1, panels A and B). Furthermore, determined concentrations from both laboratories were correlated for lithium heparin plasma $(\mathrm{r}=$ $0.98, P<0.01)$ and sodium fluoride plasma $(\mathrm{r}=0.99$, $P<0.01)$. Both laboratories measured higher L-lactate concentrations in lithium heparin plasma compared with sodium fluoride plasma (mean difference: laboratory 1: $0.27 \pm 0.3 \mathrm{mmol} / \mathrm{L}, \mathrm{n}=49, P<0.01$; laboratory 2: $0.3 \pm 0.31 \mathrm{mmol} / \mathrm{L}, \mathrm{n}=49, P<0.01$; Figure 1 , panels $\mathrm{C}$ and D). Nevertheless, in both laboratories, L-lactate concentrations measured in lithium heparin plasma and sodium fluoride plasma were correlated (laboratory 1: $\mathrm{r}=0.75, \mathrm{n}=49, P<0.01$; laboratory $2: \mathrm{r}=0.78, \mathrm{n}=49, P<0.01$; Table 2, Experiment 1).

For the second objective, the samples were analyzed within 5 min of collection with Lactate Scout and Biosen C_line and the concentrations compared with the results of laboratory 1 considering both anticoagulants. Concentrations of L-lactate measured in lithium heparin did not differ when analyzed with the Lactate Scout $(0.99 \pm 0.35 \mathrm{mmol} / \mathrm{L}, \mathrm{n}=46)$, the Biosen C_line (0.81 $\pm 0.26 \mathrm{mmol} / \mathrm{L}, \mathrm{n}=41)$, and the laboratory $(1.0 \pm$ $0.36 \mathrm{mmol} / \mathrm{L}, \mathrm{n}=48, P>0.05)$. Concentrations of L-lactate measured in sodium fluoride, however, were higher when analyzed with the Lactate Scout (1.85 $\pm 0.66 \mathrm{mmol} / \mathrm{L}, \mathrm{n}=48)$ compared with the Biosen C_line $(0.92 \pm 0.37 \mathrm{mmol} / \mathrm{L}, \mathrm{n}=35, P<0.05)$ and the commercial laboratory $(0.72 \pm 0.45 \mathrm{mmol} / \mathrm{L}, \mathrm{n}=$ 48, $P<0.05)$. Comparing both anticoagulants, values did not differ between the 3 methods except for values measured by the Lactate Scout in sodium fluoride $(P<$ 0.05 , Figure 2). The number of analyzed blood samples differed because L-lactate concentrations $<0.5 \mathrm{mmol} / \mathrm{L}$ cannot be measured by the Lactate Scout and the Biosen C_line.

In experiment 2 , blood samples from 173 dairy cows were analyzed with the Lactate Scout, the Biosen C_ line, and a commercial laboratory (laboratory 1) using lithium heparin as anticoagulant. L-Lactate concentrations were correlated between the 3 different methods (Lactate Scout vs. Biosen C_line: $\mathrm{r}=0.85, \mathrm{n}=110$, $P<0.01$; Lactate Scout vs. laboratory 1: $\mathrm{r}=0.75, \mathrm{n}$ $=163, P<0.01$; Biosen C_line vs. Laboratory $1: \mathrm{r}=$ $0.86, \mathrm{n}=120, P<0.01)$. Mean L-lactate concentrations were higher when measured by the commercial laboratory $(0.9 \pm 0.3 \mathrm{mmol} / \mathrm{L}, \mathrm{n}=173)$ compared with those measured with the Lactate Scout $(0.7 \pm 0.3 \mathrm{mmol} / \mathrm{L}$, $\mathrm{n}=163)$ and the Biosen C_line $(0.7 \pm 0.2 \mathrm{mmol} / \mathrm{L}$, $\mathrm{n}=120, P<0.05$; Figure 3, panels A to C; Table 2, Experiment 2). Again, the number of samples differed for the reason described above.

Similarly, in experiment 3, blood samples from 106 calves were analyzed using lithium heparin as anticoagulant. L-Lactate concentrations were correlated between the 3 different methods (Lactate Scout vs. Biosen C_line: $\mathrm{r}=0.97, \mathrm{n}=102, P<0.01$; Lactate Scout vs. laboratory 1: $\mathrm{r}=0.98, \mathrm{n}=105, P<0.01$; Biosen C_line vs laboratory $1: \mathrm{r}=0.99, \mathrm{n}=103, P$ $<0.01)$. Mean L-lactate concentrations did not differ between the 3 methods tested (Lactate Scout: $1.7 \pm 1.1$ $\mathrm{mmol} / \mathrm{L}, \mathrm{n}=105$; Biosen C_line: $1.4 \pm 1.1 \mathrm{mmol} / \mathrm{L}, \mathrm{n}$ $=103$; laboratory 1: $1.8 \pm 1.3 \mathrm{mmol} / \mathrm{L}, \mathrm{n}=106, P>$ 0.05; Figure 3, panels D to F; Table 2, Experiment 3). Again, the number of samples differed for the reason described above.

\section{DISCUSSION}

In experiment 1 , we tested the accuracy of measurements of plasma L-lactate concentration in 2 laboratories using lithium heparin and sodium fluoride as anticoagulants. Measurements of both laboratories were correlated and both laboratories measured $0.3 \mathrm{mmol} / \mathrm{L}$ higher plasma L-lactate concentrations in lithium heparin than in sodium fluoride. In sodium fluoride, glycolysis is inhibited, whereas in lithium heparin glycolysis continues (Astles et al., 1994). Therefore, the time lag between sampling and freezing the centrifuged samples $(60 \pm 30 \mathrm{~min})$ might explain our observation.

From a preliminary trial, we suspected that L-lactate concentrations measured with the Lactate Scout were higher in samples collected with sodium fluoride as anticoagulant rather than lithium heparin. Therefore, we determined L-lactate concentration from the same cow with the Lactate Scout, the Biosen C_line, and a commercial laboratory in each anticoagulant. Concentrations of L-lactate measured in lithium heparin did not differ when analyzed with the Lactate Scout, the Biosen C_line, and the laboratory. Concentrations of Llactate measured in sodium fluoride, however, were considerably higher when analyzed with the Lactate Scout 
A)

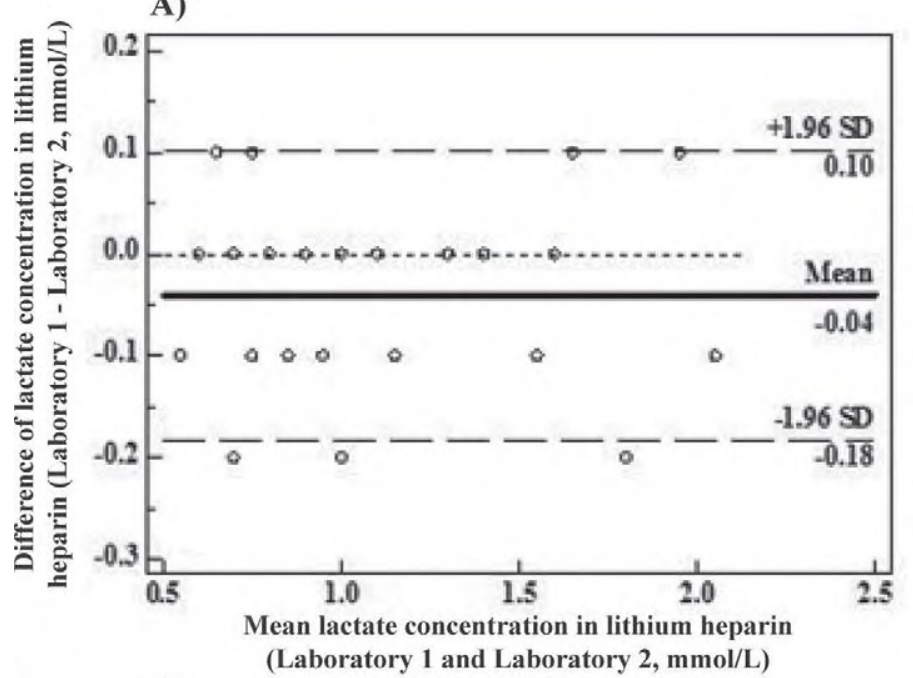

C)

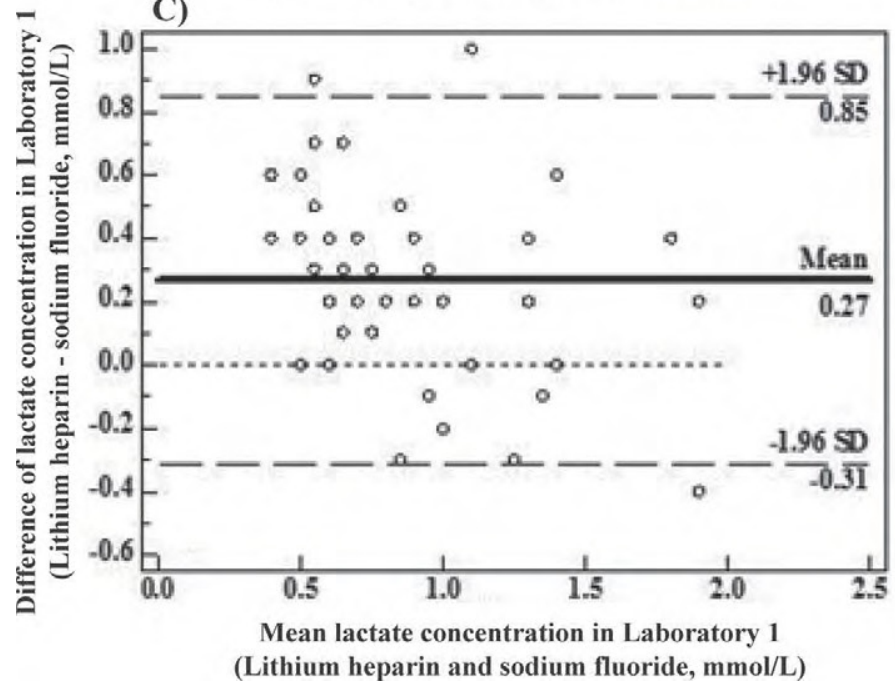

B)

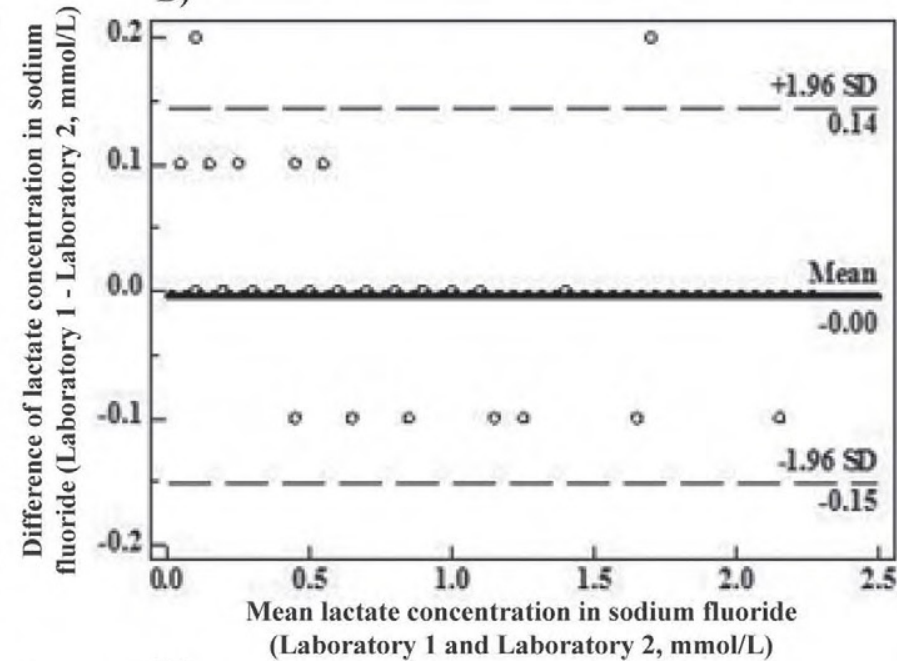

D)

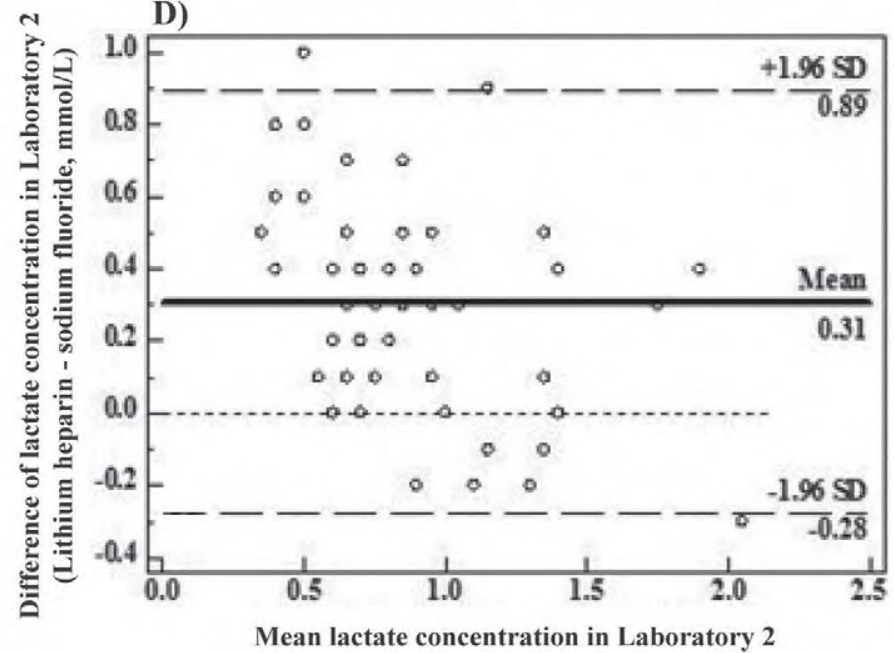

(Lithium heparin and sodium fluoride, $\mathrm{mmol} / \mathrm{L}$ )

Figure 1. Bland-Altman plots comparing blood L-lactate concentrations measured by 2 different laboratories in lithium heparin (A) and sodium fluoride (B) and comparing L-lactate concentrations measured in lithium heparin and sodium fluoride within laboratory 1 (C) and laboratory $2(\mathrm{D})$.

compared with the Biosen C_line and the commercial laboratory. Comparing both anticoagulants, values did not differ between the 3 methods except for values measured by the Lactate Scout in sodium fluoride. The reasons for the higher concentrations determined with the Lactate Scout in sodium fluoride remain unclear. However, we speculate that sodium fluoride might have influenced the electrochemical reaction. A similar interference was described for glucose measurements in diabetic patients treated with ascorbic acid or acetaminophen (Tang et al., 2000). Therefore, we do not recommend measuring L-lactate concentrations with the Lactate Scout in blood samples that use sodium fluoride as anticoagulant. However, sodium fluoride is needed as an anticoagulant when samples are processed or analyzed after a time lag to inhibit glycolysis and thus to avoid increasing lactate concentrations (Astles et al., 1994). In this case, another method could be used for analysis (i.e., Biosen C_line or commercial laboratory).

Castagnetti et al. (2010) and Ferasin et al. (2007) also validated the Lactate Scout for foals and dogs, respectively. To our knowledge, ours is the first study validating an electrochemical handheld lactate meter for cows and calves. In calves, the correlation determined between the handheld meter and the laboratory $(\mathrm{r}=0.98)$ was comparable to that reported by Coghe et al. (2000) with a portable, photometric lactate analyzer and a laboratory for calves $(\mathrm{r}=0.94)$. Furthermore, in our study, we observed no significant difference between the handheld meter and the laboratory as a reference method, whereas Coghe et al. (2000) reported an over- 
Table 2. Test characteristics of the different analytical methods tested in experiments 1,2 , and 3

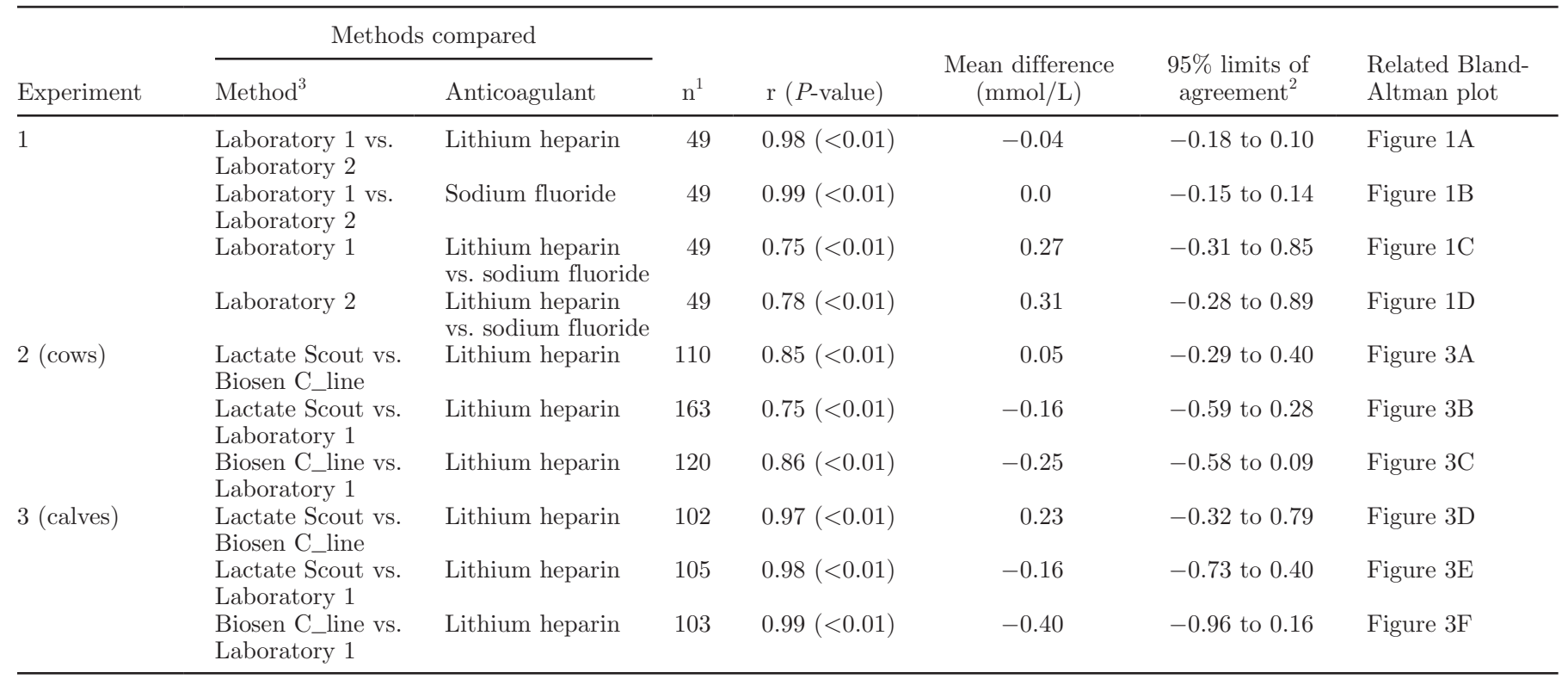

${ }^{1}$ Number of samples.

${ }^{2}$ Limits of agreement were calculated with the method of Bland and Altman (1986) as follows: mean difference $\pm 1.96 \times$ SD.

${ }^{3}$ Lactate Scout was from SensLab GmbH (Leipzig, Germany) and Biosen C_line was from EKF Diagnostics GmbH (Barleben, Germany).

estimation when using the photometric meter. In cows, however, we found a significant but clinically negligible difference between the handheld meter and the laboratory as the reference method. A possible explanation for higher L-lactate concentrations measured by the laboratory might be ongoing glycolysis between sampling and centrifuging the plasma, although samples were centrifuged within $20 \pm 10$ min after sampling and stored on ice.

Comparing correlations, the Lactate Scout seems to measure L-lactate more accurately in calves than in cows. However, the high correlation in calves in experiment 3 might be due to a higher range of L-lactate concentrations than in cows in experiment 2 (calves: 0.5
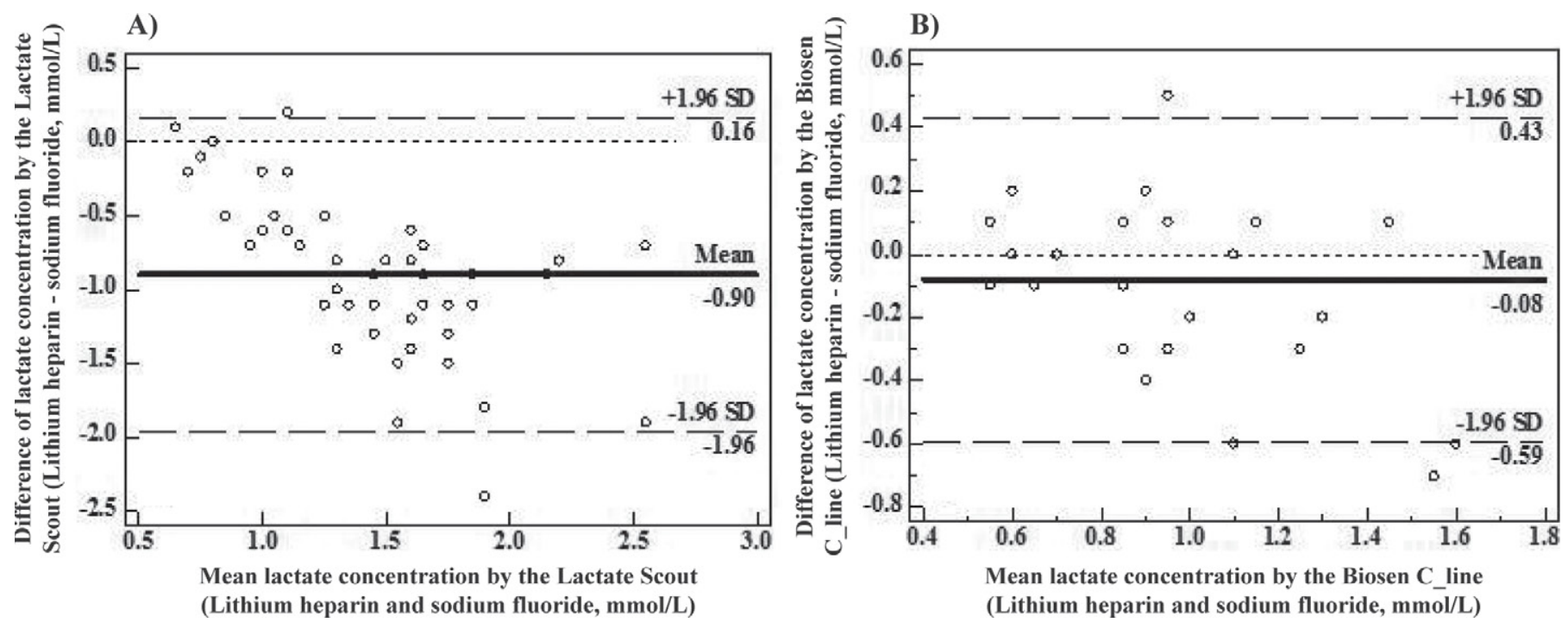

Figure 2. Bland-Altman plots comparing blood L-lactate concentrations measured in lithium heparin and sodium fluoride with the Lactate Scout (A; SensLab GmbH, Leipzig, Germany) and the Biosen C_line (B; EKF Diagnostics GmbH, Barleben, Germany). 
A)

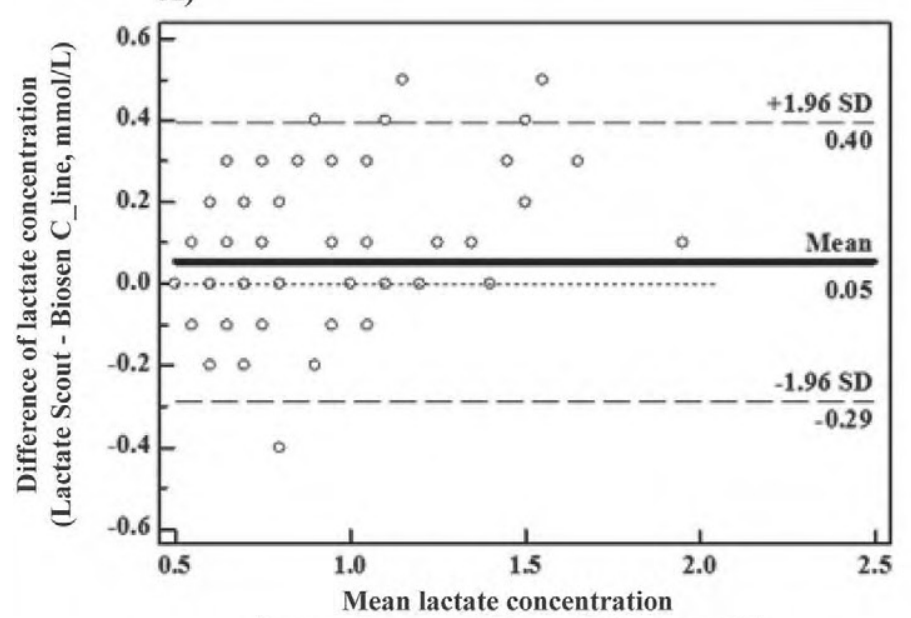

(Lactate Scout and Biosen C line, $\mathrm{mmol} / \mathrm{L}$ )

B)

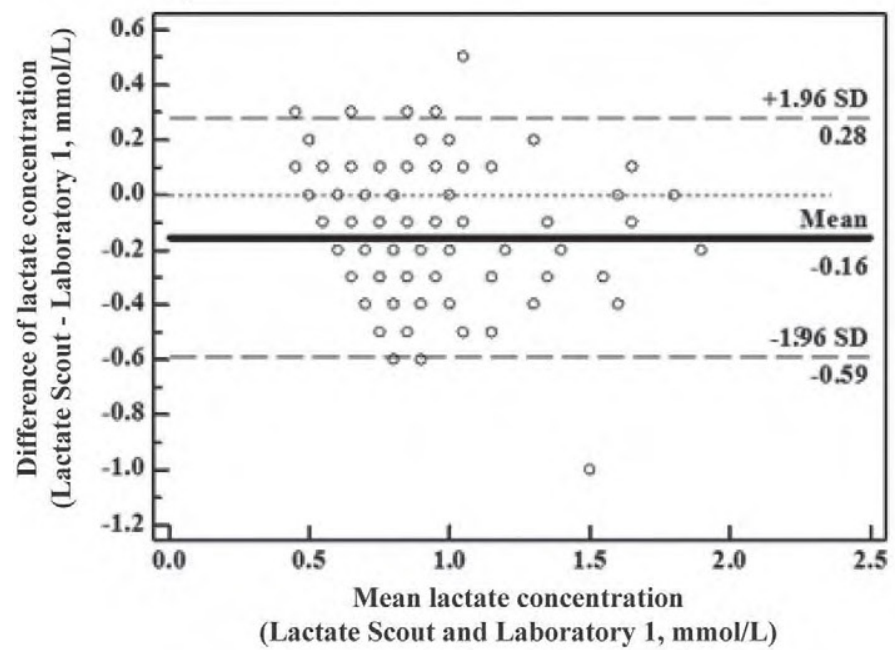

C)

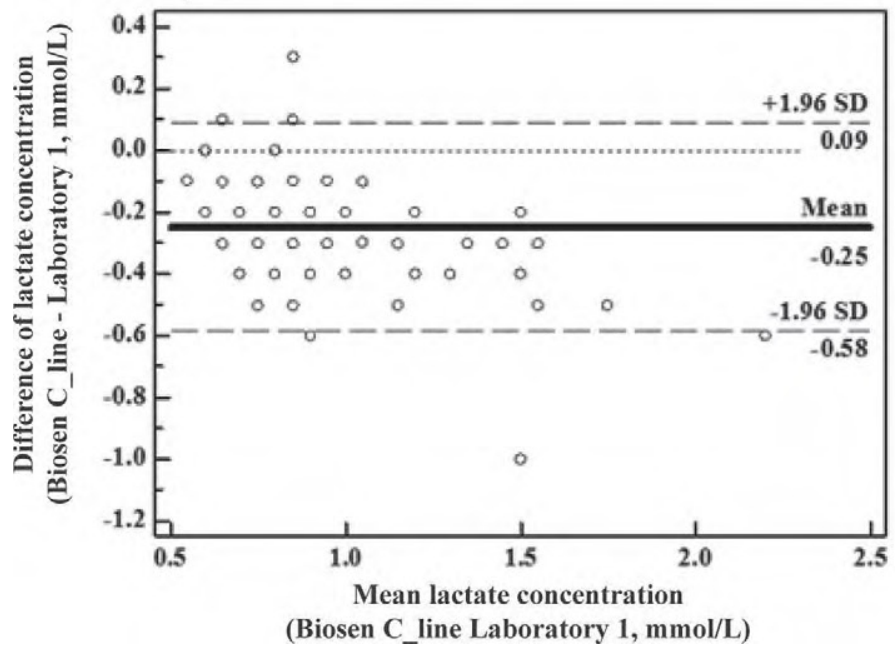

D)

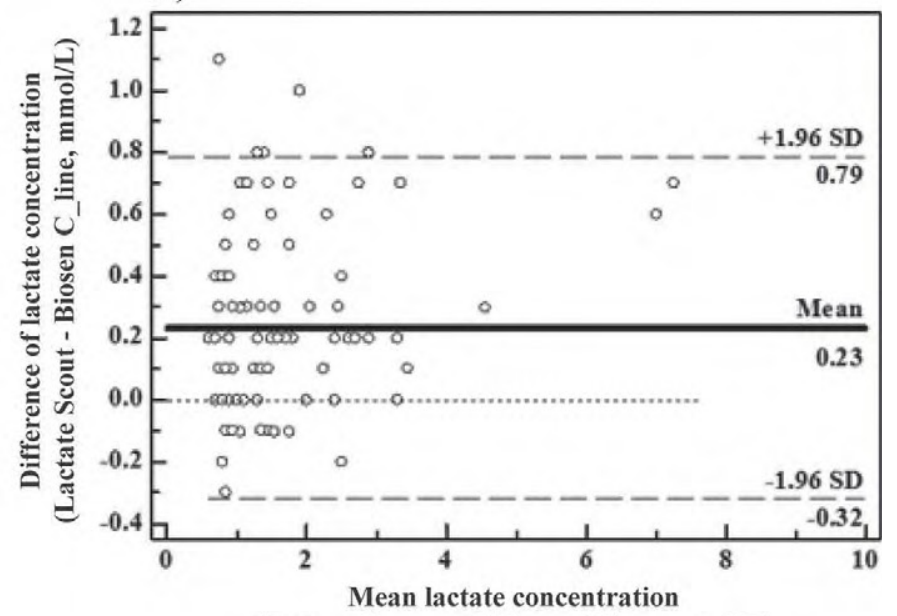

(Lactate Scout and Biosen $\mathrm{C}$ line, $\mathrm{mmol} / \mathrm{L}$ )

E)

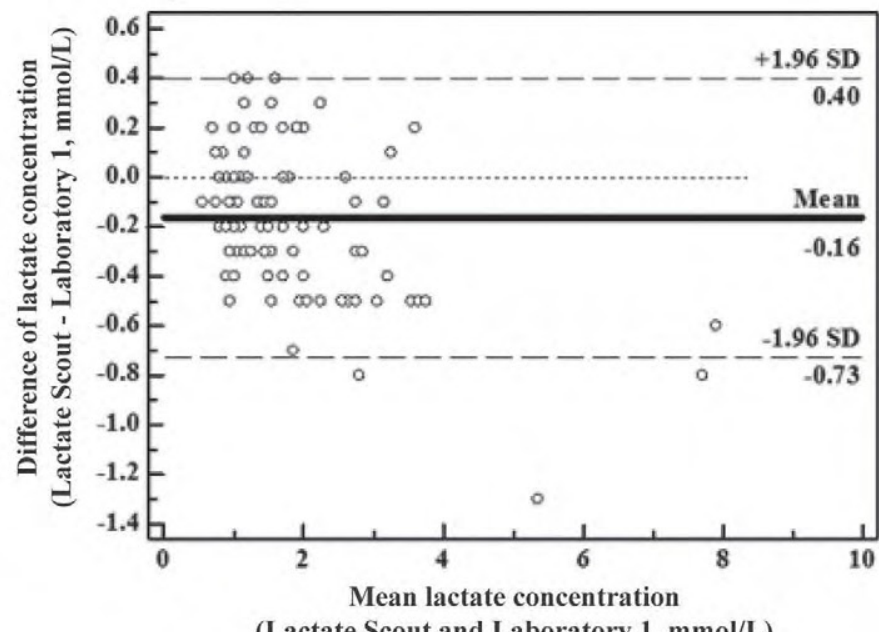

(Lactate Scout and Laboratory $1, \mathrm{mmol} / \mathrm{L}$ )

F)

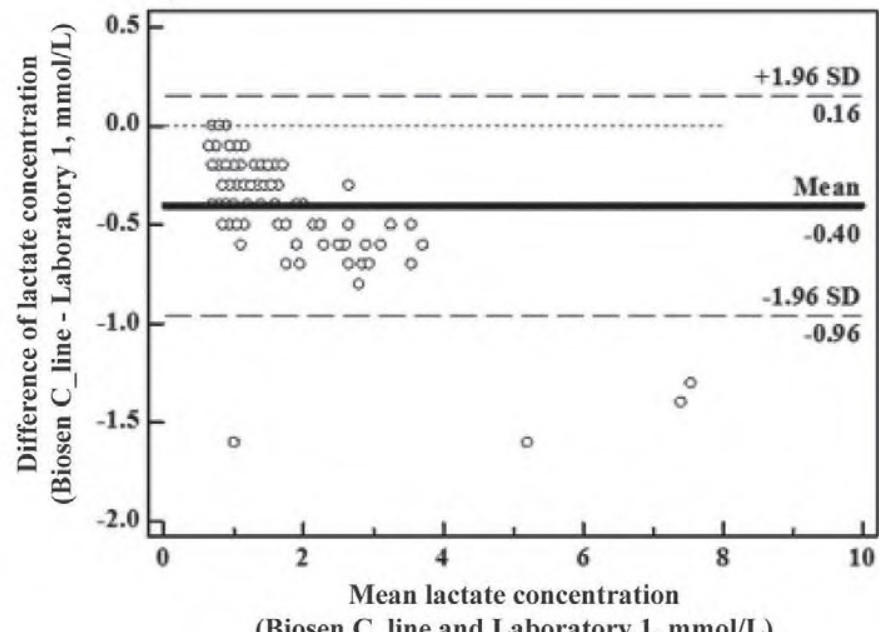

(Biosen C_line and Laboratory $1, \mathrm{mmol} / \mathrm{L}$ )

Figure 3. Bland-Altman plots comparing blood L-lactate concentrations measured with the Lactate Scout and the Biosen C_line (EKF Diagnostics GmbH, Barleben, Germany) in cows (A) and in calves (D), the Lactate Scout (SensLab GmbH, Leipzig, Germany) and laboratory 1 in cows (B) and calves (E), and the Biosen C_line and laboratory 1 in cows (C) and calves (F). 
to $7.6 \mathrm{mmol} / \mathrm{L}$; cows: 0.5 to $2.0 \mathrm{mmol} / \mathrm{L}$ ) and thus have increased the determined correlation.

\section{CONCLUSIONS}

The Lactate Scout and Biosen C_line measure blood L-lactate concentrations reliably compared with a commercial laboratory as the reference method. The Lactate Scout is a useful tool for accurate animal-side detection of blood L-lactate concentrations of cows and calves. Animal-side lactate concentrations might be of interest when studying the prognosis of different diseases in calves (Coghe et al., 2000) or when developing strategies to mitigate dystocia-related increases of plasma lactate concentrations (Diesch et al., 2004). However, attention needs to be paid to the choice of anticoagulant. On the one hand, sodium fluoride inhibits glycolysis, which is necessary when samples are processed and analyzed with a time lag. On the other hand, sodium fluoride interferes with the measurements of the Lactate Scout in terms of an overestimation of L-lactate concentration. Therefore, lithium heparin should be used as anticoagulant when determining L-lactate concentration animal-side with the Lactate Scout.

\section{ACKNOWLEDGMENTS}

We thank E. Tönniges, SensLab GmbH (Leipzig, Germany), and EKF Diagnostics GmbH (Barleben, Germany) for their kind contribution to this project in providing the Lactate Scout and the Bisosen C_line devices, respectively. Furthermore, we greatly appreciate the kind cooperation of the farm personnel on the dairy farm and thank L. Bella, S. Bonk, C. FischerTenhagen, M. Grau, A. Mahrt, R. Voigtsberger, and N. Zimmermann (Clinic for Animal Reproduction, Berlin, Germany) for their help in collecting and analyzing the blood samples.

\section{REFERENCES}

Arieff, A. I., and H. Graf. 1987. Pathophysiology of type A hypoxic lactic acidosis in dogs. Am. J. Physiol. Endocrinol. Metab. 253:271-276

Astles, R., C. P. Williams, and F. Sedor. 1994. Stability of plasma lactate in vitro in the presence of antiglycolytic agents. Clin. Chem. 40:1327-1330

Bland, J. M., and D. G. Altman. 1986. Statistical methods for assessing agreement between two methods of clinical measurement. Lancet 327:307-310.

Campbell, E. H. 2011. Lactate-driven equine conditioning programmes. Vet. J. 190:199-207.
Castagnetti, C., A. Pirrone, J. Mariella, and G. Mari. 2010. Venous blood lactate evaluation in equine neonatal intensive care. Theriogenology 73:343-357.

Coghe, J., C. H. Uystepruyst, F. Bureau, J. Detilleux, T. Art, and P. Lekeux. 2000. Validation and prognostic value of plasma lactate measurement in bovine respiratory disease. Vet. J. 160:139-146.

Davidson, J. A., and D. K. Beede. 2009. Exercise training of latepregnant and nonpregnant dairy cows affects physical fitness and acid-base homeostasis. J. Dairy Sci. 92:548-562.

Diesch, T. J., D. J. Mellor, K. J. Stafford, and R. N. Ward. 2004. The physiological and physical status of single calves at birth in a dairy herd in New Zealand. N. Z. Vet. J. 52:250-255.

Ferasin, L., S. J. Dodkin, A. Amodio, J. K. Murray, and K. Papasouliotis. 2007. Evaluation of a portable lactate analyzer (Lactate Scout) in dogs. Vet. Clin. Pathol. 36:36-39.

Guerci, B., N. Tubiana-Rufi, B. Bauduceau, R. Bresson, A. Cuperlier, C. Delcroix, D. Durain, C. Fermon, J. P. Le Floch, C. Le Devehat, V. Melki, L. Monnier, H. Mosnier-Pudar, P. Taboulet, and H. Hanaire-Broutin. 2005. Advantages to using capillary blood $\beta$-hydroxybutyrate determination for the detection and treatment of diabetic ketosis. Diabetes Metab. 31:401-406.

Hellmann, K., and I. Radeloff. 2000. Guidance for industry: Good clinical practice. International Cooperation on Harmonisation of Technical Requirements for Registration of Veterinary Medicinal Products (VICH). VICH, Brussels, Belgium. http://www.fda.gov/ downloads/AnimalVeterinary/GuidanceComplianceEnforcement/ GuidanceforIndustry/UCM052417.pdf.

Hughes, D., E. R. Rozanski, F. S. Shofer, L. L. Laster, and K. J. Drobatz. 1999. Effect of sampling site, repeated sampling, pH, and $\mathrm{pCO}_{2}$ on plasma lactate concentration in healthy dogs. Am. J. Vet. Res. 60:521-524.

Iwersen, M., U. Falkenberg, R. Voigtsberger, D. Forderung, and W. Heuwieser. 2009. Evaluation of an electronic cowside test to detect subclinical ketosis in dairy cows. J. Dairy Sci. 92:2618-2624.

Lagutchik, M. S., G. K. Ogilvie, T. B. Hackett, and W. E. Wingfield. 1998. Increased lactate concentrations in ill and injured dogs. J. Vet. Emerg. Crit. Care 8:117-127.

Lagutchik, M. S., G. K. Ogilvie, W. E. Wingfield, and T. B. Hackett. 1996. Lactate kinetics in veterinary critical care: A review. J. Vet. Emerg. Crit. Care 6:81-95.

Mizock, B. A. 1987. Controversies in lactic acidosis. Implications in critically ill patients. JAMA 258:497-501.

Mülling, M., H. Waizenhöfer, and B. Brattig. 1979. Glukose-, Laktatund $\mathrm{pH}$-Werte bei Kühen und Kälbern während und unmittelbar nach der Geburt. Berl. Munch. Tierarztl. 92:111-117.

Naylor, J. M. 1987. Severity and nature of acidosis in diarrheic calves over and under one week of age. Can. Vet. J. 28:168-173.

Sorge, U., D. Kelton, and R. Staufenbiel. 2009. Neonatal blood lactate concentration and calf morbidity. Vet. Rec. 164:533-534.

Steiss, J., H. A. Ahmad, P. Cooper, and C. Ledford. 2004. Physiologic responses in healthy Labrador Retrievers during field trial training and competition. J. Vet. Intern. Med. 18:147-151.

Tang, Z., X. Du, R. F. Louie, and G. J. Kost. 2000. Effects of drugs on glucose measurements with handheld glucose meters and a portable glucose analyzer. Am. J. Clin. Pathol. 113:75-86.

Tas, O., H. De Rooster, E. Baert, M. H. Doom, and L. Duchateau 2008. The accuracy of the Lactate Pro handheld analyser to determine blood lactate in healthy dogs. J. Small Anim. Pract. 49:504-508.

Thorneloe, C., C. Bedard, and S. Boysen. 2007. Evaluation of a handheld lactate analyzer in dogs. Can. Vet. J. 48:283-288.

Wess, G., and C. Reusch. 2000a. Assessment of five portable blood glucose meters for use in cats. Am. J. Vet. Res. 61:1587-1592.

Wess, G., and C. Reusch. 2000b. Evaluation of five portable blood glucose meters for use in dogs. J. Am. Vet. Med. Assoc. 216:203-209. 